\title{
Determinantal Formula for Generalized Riffle Shuffle
}

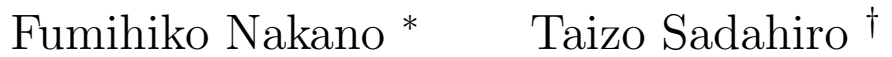

March 23, 2021

\begin{abstract}
We consider a generalized riffle shuffle on the colored permutation group $G_{p, n}$ and derive a determinantal formula for the probability of finding descents at given positions, proof of which is based on the bijection between the set of shuffles in question and that of non-intersecting lattice paths.
\end{abstract}

\section{Introduction}

There are many studies on the theory of riffle shuffling on the permutation group $S_{n}$ from both algebraic and probabilistic point of view. For instance, Bayer-Diaconis formulated the riffle shuffle as a random operation on $S_{n}$ and studied the corresponding random walk ; they analyzed the speed of convergence to the stationary distribution and showed that it exhibits the cut off phenomenon (e.g., [1]). Diaconis-Fulman [3, 4] showed that the descent process of this random walk is a Markov chain which has the same distribution as that of the carries process in adding numbers studied by Holte [7. The authors in this paper previously considered a generalized riffle shuffle on the colored permutation group $G_{p, n}$, and a generalized carries process

\footnotetext{
${ }^{*}$ Mathematical Institute, Tohoku University, Sendai 980-8578, Japan e-mail : fumihiko.nakano.e4@tohoku.ac.jp

${ }^{\dagger}$ Department of Computer Science, Tsuda Colledge, 2-1-1, Tsuda, Kodaira City, 1878577, Tokyo, Japan. e-mail : sadahiro@tsuda.ac.jp
} 
and showed that the descent process of the former has the same distribution as the latter [11, 12]. In this paper, we study the generalized riffle shuffle studied in 12 and derive a formula for the probability of finding descents at given positions. To describe our results, we introduce some notations and definitions.

First of all, we consider a set $\Sigma:=[n] \times \mathbf{Z}_{p}([n]:=\{1,2, \cdots, n\}, p \in \mathbf{N})$. For $q \in \mathbf{Z}_{p}$ let $T_{q}: \Sigma \rightarrow \Sigma$ be a shift given by $T_{q}(i, r):=(i, r+q),(i, r) \in \Sigma$. The group $G_{p, n}\left(\simeq \mathbf{Z}_{p} 2 S_{n}\right)$ of colored permutations is defined as the set of bijections on $\Sigma$ which commute with $T$ :

$$
G_{p, n}:=\left\{\sigma: \Sigma \rightarrow \Sigma \mid \sigma: \text { bijection s.t. } \sigma \circ T_{1}=T_{1} \circ \sigma\right\} .
$$

By definition, $\sigma \in G_{p, n}$ is characterized by $\left\{\left(\sigma(i), \sigma_{c}(i)\right)\right\}_{i=1}^{n}$, where $\left(\sigma(i), \sigma_{c}(i)\right):=$ $\sigma(i, 0) \in \Sigma, i \in[n]$, and henceforth we write

$$
\sigma=\left(\left(\sigma(1), \sigma_{c}(1)\right),\left(\sigma(2), \sigma_{c}(2)\right), \cdots,\left(\sigma(n), \sigma_{c}(n)\right)\right) .
$$

We define below two notions of order and corresponding descents on $G_{p, n}$.

(1) (usual order and descent)

We consider an linear order on $\Sigma$ as follows :

$$
\begin{aligned}
& (1,0)<(2,0)<\cdots<(n, 0)< \\
& (1, p-1)<(2, p-1)<\cdots<(n, p-1)< \\
& (1, p-2)<(2, p-2)<\cdots<(n, p-2)< \\
& \cdots \\
& (1,1)<(2,1)<\cdots<(n, 1) .
\end{aligned}
$$

We say that $\sigma \in G_{p, n}$ has a d-descent at $i$ if and only if (i) $\left(\sigma(i), \sigma_{c}(i)\right)>$ $\left(\sigma(i+1), \sigma_{c}(i+1)\right)$ (for $\left.i=1,2, \cdots, n-1\right)$ and (ii) $\sigma_{c}(n) \neq 0($ for $i=n)$. We denote by $d(\sigma)$ the number of descents of $\sigma$.

(2) (dash-order and dash-descent)

We also consider another notion of order and corresponding descent. We define the "dash-order" $<$ on $\Sigma$ :

$$
\begin{aligned}
& (1,0)<^{\prime}(2,0)<^{\prime} \cdots<^{\prime}(n, 0)<^{\prime} \\
& (1,1)<^{\prime}(2,1)<^{\prime} \cdots<^{\prime}(n, 1)<^{\prime} \\
& \cdots \\
& (1, p-1)<^{\prime}(2, p-1)<^{\prime} \cdots<^{\prime}(n, p-1) .
\end{aligned}
$$


we say that $\sigma \in G_{p, n}$ has a d'-descent at $i$ if and only if (i') $\left(\sigma(i), \sigma_{c}(i)\right)>^{\prime}$ $\left(\sigma(i+1), \sigma_{c}(i+1)\right)$ (for $i=1,2, \cdots, n-1$ ) and (ii') $\sigma_{c}(n)=p-1$ (for $i=n)$. We denote by $d^{\prime}(\sigma)$ the number of dash-descents of $\sigma$. For $p=2$, $d^{\prime}(\sigma)=d(\sigma)$.

Here we shall consider the generalized riffle shuffle called $(b, n, p)$-shuffle which is, roughly speaking, to carry out the usual $b$-shuffle to " $n$ cards with $p$ colors", but to apply $T_{r}$-shift to the $(j p+r)$-th pile. To be precise, it is defined as follows :

(i) pick up $n$ numbers uniformly at random from $\mathcal{D}(b):=\{0,1, \cdots, b-1\}$ yielding an array of numbers $\mathbf{A}:=\left(A_{1}, A_{2}, \cdots, A_{n}\right) \in \mathcal{D}(b)^{n}$, which we call labels,

(ii) rearrange $1,2, \cdots, n$ according to the order of labels $A_{1}, \cdots, A_{n}$ so that for each $i=1, \cdots, n$ we have $k_{i} \in[n] 1$. This is the same procedure to have the $b$-shuffle from its Gilbert-Shanon-Reeds representation (GSR representation in short),

(iii) If $a_{i} \equiv q_{i} \in \mathbf{Z}_{p}(\bmod p)$, set $\left(\sigma(i), \sigma_{c}(i)\right):=\left(k_{i}, q_{i}\right), i=1, \cdots, n$, which determines $\sigma=\left(\left(\sigma(1), \sigma_{c}(1)\right),\left(\sigma(2), \sigma_{c}(2)\right), \cdots,\left(\sigma(n), \sigma_{c}(n)\right)\right) \in G_{p, n}$. We denote $\sigma$ by $\pi[\mathbf{A}] \in G_{p, n}$, and call $\mathbf{A}=\left(A_{1}, \cdots, A_{n}\right)$ the GSR representation of $\sigma$. We show an example below. In this example, $(b, n, p)=(5,4,3)$, and $\mathbf{A}=(4,0,2,4) \in \mathcal{D}(5) . \sigma$ has d-descents at $i=1,4$, and d'- descents at $i=1,3$.

\begin{tabular}{c|cc}
$i$ & $A_{i}$ & $\left(\sigma(i), \sigma_{c}(i)\right)$ \\
\hline 1 & 4 & $(3,1)$ \\
2 & 0 & $(1,0)$ \\
3 & 2 & $(2,2)$ \\
4 & 4 & $(4,1)$
\end{tabular}

We shall consider the probability that $(b, n, p)$-shuffle has d-descents or d'descents at given positions. Let $k \in[n]$, take $s_{1}, \cdots, s_{k}$ such that $0<s_{1}<$ $s_{2}<\cdots<s_{k} \leq n$, and let $s_{0}:=0, s_{k+1}:=n$. We set :

$$
\begin{aligned}
P\left(s_{1}, \cdots, s_{k}\right) & :=\mathbf{P}\left((b, n, p) \text {-shuffle has d-descents } \operatorname{at}\left(s_{1}, \cdots, s_{k}\right)\right) \\
P^{\prime}\left(s_{1}, \cdots, s_{k}\right) & :=\mathbf{P}\left((b, n, p) \text {-shuffle has d'-descents at }\left(s_{1}, \cdots, s_{k}\right)\right) .
\end{aligned}
$$

\footnotetext{
${ }^{1}$ To be explicit, we first arrange $A_{1}, \cdots, A_{n}$ in ascending order, say $A_{i(1)}, A_{i(2)}, \cdots, A_{i(n)}$. Then we set $\sigma^{-1}(1)=i(1), \cdots, \sigma^{-1}(n)=i(n)$.
} 


\section{Theorem 1.1.}

Let $b \equiv 1(\bmod p)$ so that $b=p c+1$ for some $c \in \mathbf{N}$. Then $P\left(s_{1}, s_{2}, \cdots, s_{k}\right)$ is given by the following determinantal formula:

(1) $s_{k}<n$ :

$$
\begin{aligned}
& P\left(s_{1}, \cdots, s_{k}\right)= \frac{1}{b^{n}} \operatorname{det}(f(i, j))_{i, j=0, \cdots, k} \\
& \text { where } f(i, j):= \begin{cases}\left(\begin{array}{c}
s_{j+1}-s_{i}+b-1 \\
b-1 \\
b-s_{i}+c \\
c
\end{array}\right), & (0 \leq i \leq k, 0 \leq j \leq k-1) \\
\left.\left(\begin{array}{c}
n
\end{array}\right), i \leq k, j=k\right)\end{cases}
\end{aligned}
$$

(2) $s_{k}=n$ :

$$
P\left(s_{1}, \cdots, s_{k-1}, n\right)=\frac{1}{b^{n}} \operatorname{det}(f(i, j))_{i, j=0, \cdots, k-1}
$$

$$
\text { where } f(i, j):= \begin{cases}\left(\begin{array}{c}
s_{j+1}-s_{i}+b-1 \\
b-1 \\
n-s_{i}+b-1 \\
b-1
\end{array}\right)-\left(\begin{array}{c}
n-s_{i}+c \\
c
\end{array}\right), \quad(0 \leq i \leq k-1, j=k-1) & (0 \leq i \leq k-1,0 \leq j \leq k-2)\end{cases}
$$

\section{Example 1}

Let $p=4, c=2, n=6, b=4 \cdot 2+1=9$, and $k=3$.

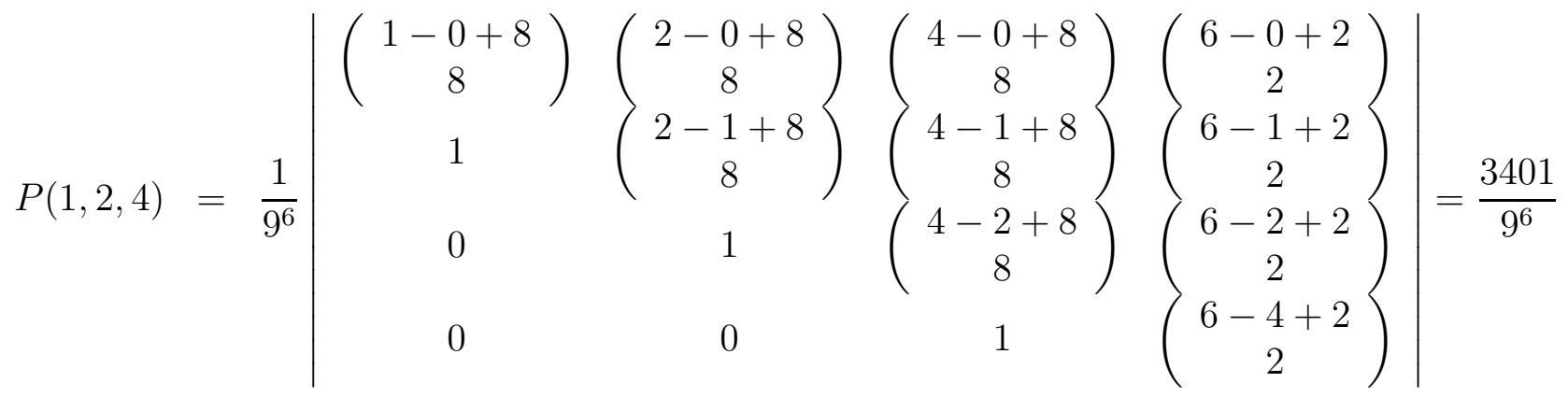

$$
\begin{aligned}
& P(1,2,6)=\frac{1}{9^{6}}\left(\begin{array}{c}
\left(\begin{array}{c}
1-0+8 \\
8
\end{array}\right) \\
1 \\
0 \\
1 \\
8 \\
8 \\
2+8
\end{array}\right) \quad\left(\begin{array}{c}
2-0+8 \\
6-2+8 \\
8
\end{array}\right)-\left(\begin{array}{c}
6-0+8 \\
8 \\
6-1+8 \\
8 \\
6-2+2 \\
2
\end{array}\right)-\left(\begin{array}{c}
6-0+2 \\
2 \\
6-1+2 \\
2 \\
6
\end{array}\right)=\frac{8861}{9^{6}}
\end{aligned}
$$




\section{Remark 1.2.}

(1) These formulas have been obtained by MacMahon [9], Borodin-Diaconis-

Fulman [2] for $p=1$, and Reiner [13] for $p=2$.

(2) Let $\mathcal{L}\left(\subset \mathbf{Z}^{2}\right)$ be a subgraph of the square lattice and let $P_{0}, \cdots, P_{k}, Q_{1}, \cdots, Q_{k+1} \in$ $\mathcal{L}$ be $2(k+1)$ vertices in $\mathcal{L}$ given by

$$
\begin{aligned}
\mathcal{L} & :=(\{0,1, \cdots, n-1\} \times\{0,1, \cdots, b-1\}) \cup\{(n, j) \mid j=0,1, \cdots, c\} \\
P_{i} & :=\left(s_{i}, 0\right), \quad 0 \leq i \leq k \\
Q_{j} & :=\left(s_{j}, b-1\right), \quad 1 \leq j \leq k, \quad Q_{k+1}:=\left(s_{k+1}, c\right)
\end{aligned}
$$

Then the determinant in the formula in Theorem 1.1(1) is equal to the number of non-intersecting $(k+1)$ paths from $P_{i}$ to $Q_{i+1}(i=0,1, \cdots, k)$ of minimal length (Figure 1).

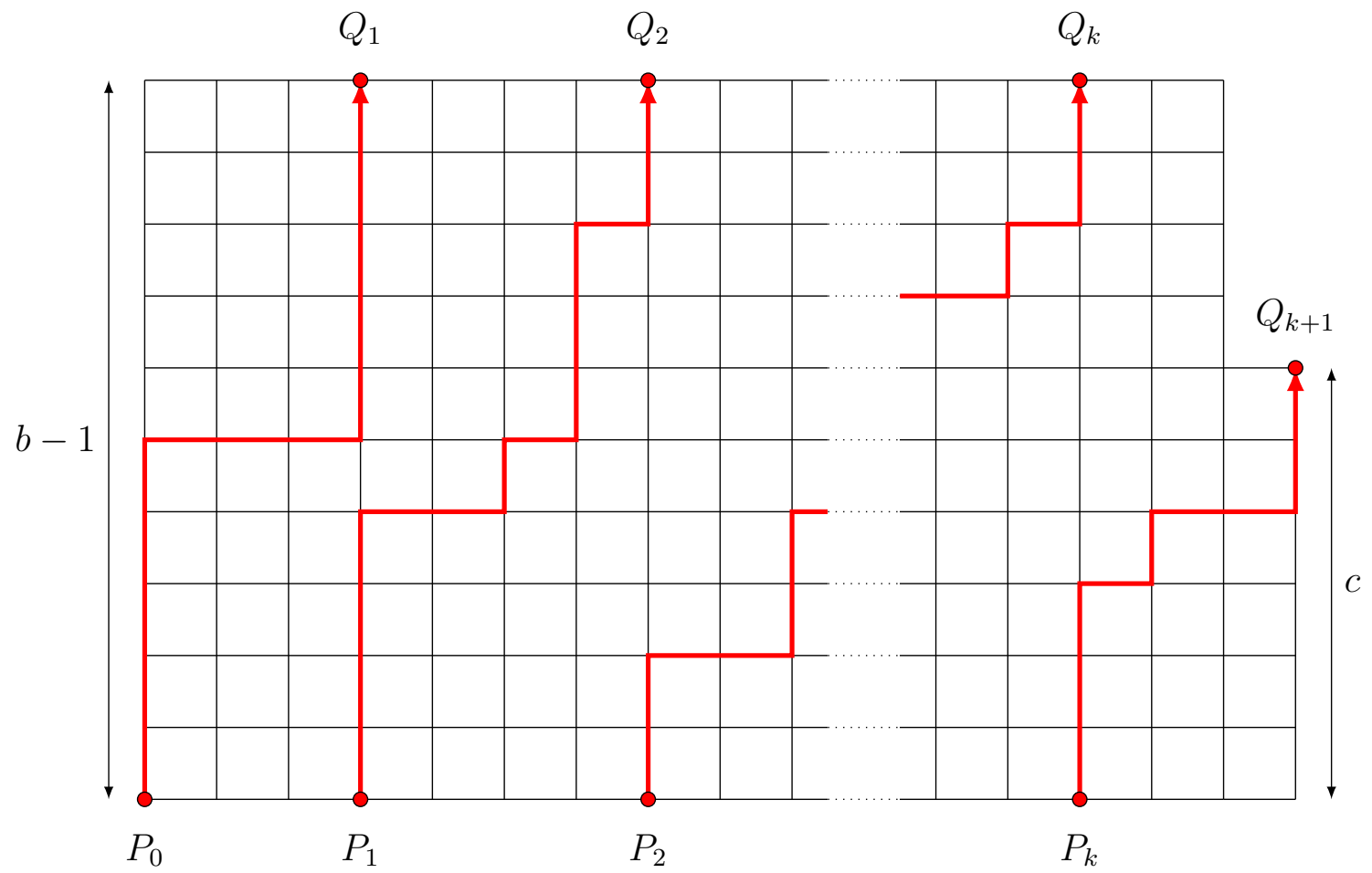

Figure 1: Non-intersecting lattice paths on $\mathcal{L}$ 
We turn to consider the case for $b \equiv-1(\bmod p)$ where we have a formula for d'-descent :

\section{Theorem 1.3.}

$b \equiv-1(\bmod p)$ so that $b=p c-1$ for some $c \in \mathbf{N} . P^{\prime}\left(s_{1}, s_{2}, \cdots, s_{k}\right)$ satisfies the same formula as Theorem 1.1 except that $f(i, j)$ is replaced by $f^{\prime}(i, j)$ given below:

(1) $s_{k}<n:$

$$
f^{\prime}(i, j):=\left\{\begin{array}{cl}
\left(\begin{array}{c}
s_{j+1}-s_{i}+b-1 \\
b-1 \\
n-s_{i}+b-c \\
b-c
\end{array}\right), & (0 \leq i \leq k, 0 \leq j \leq k-1) \\
(0 \leq i \leq k, j=k)
\end{array}\right.
$$

(2) $s_{k}=n$ :

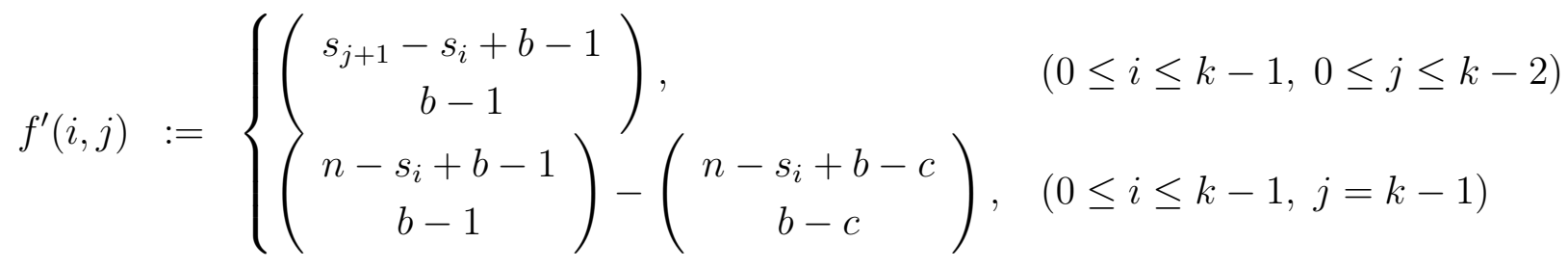

We next consider the uniform distribution on $G_{p, n}$. Since $(b, n, p)$-shuffle converges to the uniform distribution as $b \rightarrow \infty$, Theorems 1.1, 1.3, together with the equation $\left(\begin{array}{c}s+b \\ b\end{array}\right) \stackrel{b \rightarrow \infty}{=} \frac{b^{s}}{s !}\left(1+O\left(b^{-1}\right)\right)$, yield the following corollary.

\section{Corollary 1.4 .}

Let $s_{1}, \cdots, s_{k}$ with $0=s_{0}<s_{1}<\cdots<s_{k} \leq n$. The probability $P_{\text {unif }}\left(s_{1}, s_{2}, \cdots, s_{k}\right)$

(resp. $P_{\text {unif }}^{\prime}\left(s_{1}, s_{2}, \cdots, s_{k}\right)$ ) of finding the d-descents (resp. d'-descents) at $s_{1}, \cdots, s_{k}$ under the uniform distribution on $G_{p, n}$ are given respectively as follows.

(1) d-descent : 
(i) $s_{k}<n$ :

$$
\begin{aligned}
& P_{\text {unif }}\left(s_{1}, \cdots, s_{k}\right)=\frac{1}{b^{n}} \operatorname{det}(g(i, j))_{i, j=0, \cdots, k} \\
& \text { where } g(i, j):= \begin{cases}\frac{1}{\left(s_{j+1}-s_{i}\right) !}, & (0 \leq i \leq k, 0 \leq j \leq k-1) \\
\frac{1}{\left(s_{j+1}-s_{i}\right) !} \cdot\left(\frac{1}{p}\right)^{s_{j+1}-s_{i}}, & (0 \leq i \leq k, j=k)\end{cases}
\end{aligned}
$$

(ii) $s_{k}=n$ :

$$
\begin{aligned}
& P_{\text {unif }}\left(s_{1}, \cdots, s_{k-1}, n\right)=\frac{1}{b^{n}} \operatorname{det}(g(i, j))_{i, j=0, \cdots, k-1} \\
& \text { where } g(i, j):= \begin{cases}\frac{1}{\left(s_{j+1}-s_{i}\right) !}, & (0 \leq i \leq k-1,0 \leq j \leq k-2) \\
\frac{1}{\left(s_{j+1}-s_{i}\right) !} \cdot\left(1-\left(\frac{1}{p}\right)^{s_{j+1}-s_{i}}\right), & (0 \leq i \leq k-1, j=k-1)\end{cases}
\end{aligned}
$$

(2) d'-descent : let $p^{*}$ be the dual exponent of $p: \frac{1}{p}+\frac{1}{p^{*}}=1$.

$P_{\text {unif }}^{\prime}\left(s_{1}, \cdots, s_{k}\right)$ satisfies the same formulas as for (1) except that $g(i, j)$ is replaced by $g^{\prime}(i, j)$ given below :

(i) $s_{k}<n$ :

$$
g^{\prime}(i, j):= \begin{cases}\frac{1}{\left(s_{j+1}-s_{i}\right) !}, & (0 \leq i \leq k, 0 \leq j \leq k-1) \\ \frac{1}{\left(s_{j+1}-s_{i}\right) !} \cdot\left(\frac{1}{p^{*}}\right)^{s_{j+1}-s_{i}}, & (0 \leq i \leq k, j=k)\end{cases}
$$

(2) $s_{k}=n$ :

$$
g^{\prime}(i, j):= \begin{cases}\frac{1}{\left(s_{j+1}-s_{i}\right) !}, & (0 \leq i \leq k-1,0 \leq j \leq k-2) \\ \frac{1}{\left(s_{j+1}-s_{i}\right) !} \cdot\left(1-\left(\frac{1}{p^{*}}\right)^{s_{j+1}-s_{i}}\right), & (0 \leq i \leq k-1, j=k-1)\end{cases}
$$

The main ingredient of proof of Theorem 1.1 is to construct a bijection between the set of shuffles with given descent set and that of the nonintersecting lattice paths on $\mathcal{L}$ mentioned in Remark 1.2 (2). Then formulas 
in Theorems 1.1, 1.3 follows from Lindeström-Gessel-Viennot lemma [8, 6]. This bijection is the same as that between a generalized carries process called $(b, n, p)$-carries process, and the descent process of the random walk generated by $(b, n, p)$-shuffle, discussed in [12]. The contents of later sections are outlined as follows. In section 2, we prove theorems 1.1, 1.3. In Appendix, we introduce $(b, n, p)$-carries process and discuss some of its properties to supplement our previous works [11, 12] : (i) a simplified proof for the diagonalization of the transition probability matrix, (ii) the relation between the eigenspace of the transition probability matrix for the random walk mentioned above and the right eigenvectors of that of $(b, n, p)$-carries process, and, (iii) we study the convergence speed of this random walk and discuss the cut off phenomenon.

\section{Proof of Theorems}

We first give the proof for $b \equiv 1(\bmod p)$ so that we set $b=p c+1, c \in \mathbf{N}$. The argument of proof for $b \equiv-1(\bmod p)$ is similar, and we only mention the modifications in subsection 2.3.

\section{$2.1 s_{k}<n$}

We first consider the case for $s_{k}<n$. Our bijection between the set of $(b, n, p)$-shuffle with given position of descent and that of non-intersecting lattice paths on $\mathcal{L}$ is a composition of a couple of order preserving bijections between some ordered sets. The overall view is :

$\Pi\left(s_{1}, \cdots, s_{k}\right) \quad \longrightarrow \mathcal{D}_{b}\left(s_{1}, \cdots, s_{k}\right) \quad \stackrel{f}{\longrightarrow} \widetilde{\mathcal{D}_{b}}\left(s_{1}, \cdots, s_{k}\right) \stackrel{G S R}{\longrightarrow} S\left(s_{1}, \cdots, s_{k}\right)$

where

(i) $\Pi\left(s_{1}, \cdots, s_{k}\right)$ : set of non-intersecting lattice paths on $\mathcal{L}$ from $P_{i}$ to $Q_{i+1}$ $(i=0,1, \cdots, k)$ each of which has miminal length.

(ii) $\mathcal{D}_{b}\left(s_{1}, \cdots, s_{k}\right)$ : set of $n$ ordered elements in the ordered set $(\mathcal{D}(b),<)$ with descents at $s_{1}, \cdots, s_{k},(\mathcal{D}(b):=\{0,1, \cdots, b-1\})$

(iii) $\widetilde{\mathcal{D}_{b}}\left(s_{1}, \cdots, s_{k}\right)$ : set of $n$ ordered elements in $(\mathcal{D}(b), \prec)$ with another order $\prec$, with "tilde-descent" at $s_{1}, \cdots, s_{k}$ (order $\prec$ and tilde-descent are defined later).

(iv) $S\left(s_{1}, s_{2}, \cdots, s_{k}\right)$ : set of $(b, n, p)$-shuffle with d-descents at $\left(s_{1}, \cdots, s_{k}\right)$. 
Once we construct this bijection, Theorem 1.1 follows directly from Lindeström-Gessel-Viennot lemma [8, 6].

\section{Orders and descents}

We introduce two notions of orders and corresponding descents below.

(1) (normal ones)

We say that $\mathbf{X}=\left(X_{1}, X_{2}, \cdots, X_{n}\right) \in \mathcal{D}(b)^{n}$ has a descent at $k \in[n]$ if and only if (i) $X_{k}>X_{k+1}$ (for $k=1,2, \cdots, n-1$ ), (ii) $X_{n}>c($ for $k=n$ ).

We define the descent set of $\mathbf{X} \in \mathcal{D}(b)^{n}$ and the set of $\mathbf{X} \in \mathcal{D}(b)^{n}$ whose descent set coincides with the given positions $\left(s_{1}, \cdots, s_{k}\right)$, respectively.

$$
\begin{aligned}
D(\mathbf{X}) & :=\{k \in[n] \mid \mathbf{X} \text { has a descent at } k\}, \quad \mathbf{X} \in \mathcal{D}(b)^{n} \\
\mathcal{D}_{b}\left(s_{1}, \cdots, s_{k}\right) & :=\left\{\mathbf{X} \in \mathcal{D}(b)^{n} \mid D(\mathbf{X})=\left(s_{1}, \cdots, s_{k}\right)\right\}, \quad 1 \leq s_{1}<s_{2}<\cdots<s_{k} \leq n
\end{aligned}
$$

(2) (tilde order and tilde descent)

We consider another order $\prec$ on $\mathcal{D}(b)$. We write $x=(j, r)$ if $x=j p+r \in$ $\mathcal{D}(b)$, where $r=0,1, \cdots, p-1$ and $j= \begin{cases}0,1, \cdots, c-1 & (r=1,2, \cdots, p-1) \\ 0,1, \cdots, c & (r=0)\end{cases}$ and set

$$
\begin{aligned}
& (0,0) \prec(1,0) \prec \cdots \prec(c-1,0) \prec(c, 0) \prec \\
& (0, p-1) \prec(1, p-1) \prec \cdots \prec(c-1, p-1) \prec \\
& (0, p-2) \prec(1, p-2) \prec \cdots \prec(c-1, p-2) \prec \\
& \prec \cdots \prec \\
& (0,1) \prec(1,1) \prec \cdots \prec(c-1,1)
\end{aligned}
$$

Remark 2.1. The motivation to consider this order is the fact that the corresponding descent set of $\mathbf{A}=\left(A_{1}, \cdots, A_{n}\right) \in \mathcal{D}(b)^{n}$ coincides with the $d$ descent set of $\pi[\mathbf{A}]$ [12].

We say that $\mathbf{A}=\left(A_{1}, \cdots, A_{n}\right) \in \mathcal{D}(b)^{n}$ has a tilde-descent at $k \in[n]$ if and only if (i) $A_{k} \succ A_{k+1}($ for $k=1,2, \cdots, n-1)$, (ii) $A_{n} \not \equiv 0(\bmod p)$ (for $k=n$ ). The descent set and that of $\mathbf{A}$ 's with tilde-descents at given positions are defined by

$$
\begin{aligned}
\widetilde{D}(\mathbf{A}) & :=\{k \in[n] \mid \mathbf{A} \text { has a tilde-descent at } k \in[n]\}, \quad \mathbf{A} \in \mathcal{D}(b)^{n} \\
\widetilde{\mathcal{D}}_{b}\left(s_{1}, \cdots, s_{k}\right) & :=\left\{\mathbf{A} \in \mathcal{D}(b)^{n} \mid \widetilde{D}(\mathbf{A})=\left(s_{1}, \cdots, s_{k}\right)\right\}, \quad 1 \leq s_{1}<s_{2}<\cdots<s_{k} \leq n
\end{aligned}
$$




\section{Order-preserving bijections}

We construct the order-preserving bijections between those sets defined above.

(1) $\Pi\left(s_{1}, \cdots, s_{k}\right) \rightarrow \mathcal{D}_{b}\left(s_{1}, \cdots, s_{k}\right):$

For an element in $\Pi\left(s_{1}, \cdots, s_{k}\right)$, we set the corresponding element in $\mathcal{D}(b)^{n}$, for instance,

Example $2 p=3, c=2, b=3 \cdot 2+1=7, n=5, k=2, s_{1}=2, s_{2}=4$ :

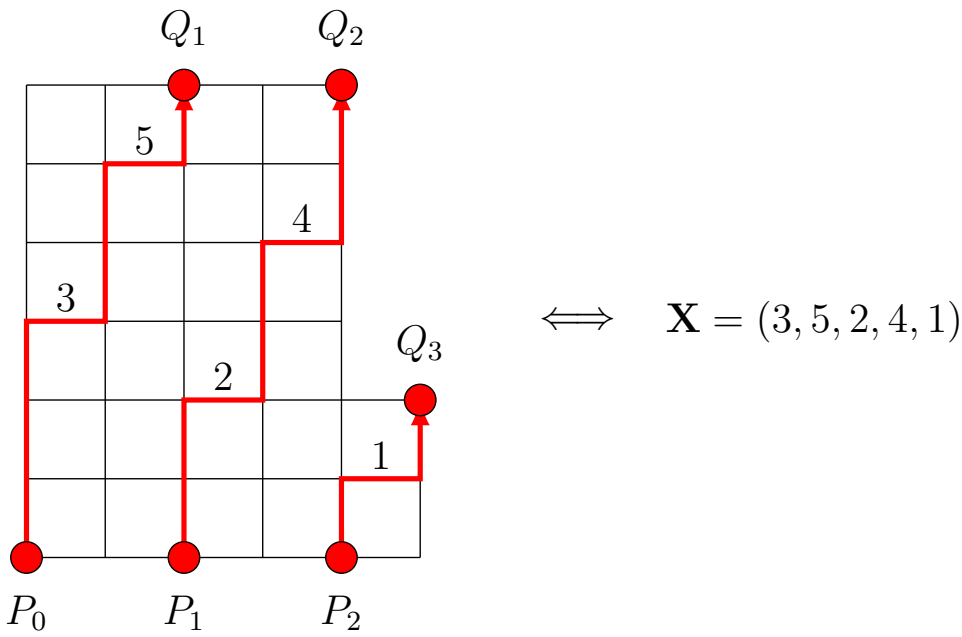

Figure 2: Bijection between $\Pi(2,4)$ and $\mathcal{D}_{7}(2,4)$

In general, for each lattice path we pick up the edges of horizontal direction and write down the heights of them. By definition this is a bijection between $\Pi\left(s_{1}, \cdots, s_{k}\right)$ and $\mathcal{D}_{b}\left(s_{1}, \cdots, s_{k}\right)$.

(2) $\mathcal{D}_{b}\left(s_{1}, \cdots, s_{k}\right) \rightarrow \widetilde{\mathcal{D}}_{b}\left(s_{1}, \cdots, s_{k}\right)$ : Let

$$
f(x) \equiv p x \quad(\bmod b) .
$$

Then [12] $f$ is a bijection on $\mathcal{D}(b)$ and (i) $x<y$ if and only if $f(x) \prec f(y)$, and (ii) $x>c$ if and only if $f(x) \not \equiv 0(\bmod p)$. Therefore $f$ is a bijection between $\mathcal{D}_{b}\left(s_{1}, \cdots, s_{k}\right)$ and $\widetilde{\mathcal{D}}_{b}\left(s_{1}, \cdots, s_{k}\right)$.

(3) $\widetilde{\mathcal{D}}_{b}\left(s_{1}, \cdots, s_{k}\right) \rightarrow S\left(s_{1}, \cdots, s_{k}\right)$ : by Remark 2.1, GSR representation gives this bijection. 
Example 2 (continued) As an example, we derive the $(b, n, p)$-shuffle corresponding to the lattice paths in Example 2. The corresponding element in $\mathcal{D}_{b}\left(s_{1}, s_{2}\right)$ is $\mathbf{X}=(3,5,2,4,1)$ and other steps are given below, all of which have descents at 2,4 .

$\begin{array}{llllll}3 & & 2 & & (2,2) \\ 5 & & & & (1,1) \\ 2 & \times 3 \stackrel{(\bmod 7)}{\longrightarrow} & 1 & 6 & \text { GSR } & (5,0) \\ 4 & & 5 & & (4,2) \\ 1 & & & & (3,0)\end{array}$

Remark 2.2. Borodin-Diaconis-Fulman [2] considered the case for $p=1$ and proved Theorem 1.1(1) in this case without using LGV lemma. Their argument can also be exploited in our situation and yields that the number of elements in $\mathcal{D}_{b}\left(s_{1}, \cdots, s_{k}\right)$ is equal to the determinant given in Theorem $1.1(1)$.

\section{$2.2 \quad s_{k}=n$}

The bijections we made in the previous subsection are effective also in this case except that of $\Pi\left(s_{1}, \cdots, s_{k}\right) \rightarrow \mathcal{D}_{b}\left(s_{1}, \cdots, s_{k}\right)$, so that it suffices to consider a relation between $\Pi\left(s_{1}, \cdots, s_{k}\right)$ and $\mathcal{D}_{b}\left(s_{1}, \cdots, s_{k}\right)$.

$$
\begin{aligned}
\mathcal{D}_{b}\left(s_{1}, \cdots, s_{k-1}, n\right)= & \left\{\mathbf{X} \in \mathcal{D}(b)^{n} \mid \mathbf{X} \text { has desents at } s_{1}, \cdots, s_{k-1} \text { and } X_{n} \geq c+1\right\} \\
=: & S_{1} \backslash S_{2} \\
\text { where } S_{1}:= & \left\{\mathbf{X} \in \mathcal{D}(b)^{n} \mid \mathbf{X} \text { has descents at } s_{1}, \cdots, s_{k-1}\right\} \\
S_{2}:= & \left\{\mathbf{X} \in \mathcal{D}(b)^{n} \mid \mathbf{X} \text { has desents at } s_{1}, \cdots, s_{k-1} \text { and } X_{n} \leq c\right\}
\end{aligned}
$$

We note that $P\left(S_{1}\right)=P\left(s_{1}, \cdots, s_{k-1}\right)$ for $p=1$ (that is, $P\left(S_{1}\right)$ is equal to the probability of finding descents at $s_{1}, \cdots, s_{k}$ for $(b, n, 1)$-shuffle) and $P\left(S_{2}\right)=P\left(s_{1}, \cdots, s_{k-1}\right)$ both of which are computed by Theorem 1.1(1). Therefore the probability in question satisfies the same determinantal formula except that the size of which is now reduced to $k \times k$ and that the last column is replaced by

$$
\left(\begin{array}{c}
n-s_{i}+b-1 \\
b-1
\end{array}\right)-\left(\begin{array}{c}
n-s_{i}+c \\
c
\end{array}\right), \quad 0 \leq i \leq k-1
$$




\section{$2.3 \quad b \equiv-1(\bmod p)$}

The essential ingredient of the proof for $b \equiv-1(\bmod p)$ is the same as that for the previous case but we modify the definition of descent, tilde-order, and tilde-descent.

(1)' (normal ones)

We say that $\mathbf{X}=\left(X_{1}, X_{2}, \cdots, X_{n}\right) \in \mathcal{D}(b)^{n}$ has a descent' at $k \in[n]$ if and only if (i) $X_{k}>X_{k+1}(k=1,2, \cdots, n-1)$, and (ii) $X_{n}>b-c$. The corresponding descent' set and the set of X's with given descent' are defined respectively by

$$
\begin{aligned}
D^{\prime}(\mathbf{X}) & :=\{k \in[n] \mid \mathbf{X} \text { has a descent' at } k \in[n]\}, \quad \mathbf{X} \in \mathcal{D}(b)^{n} \\
\mathcal{D}_{b}^{\prime}\left(s_{1}, \cdots, s_{k}\right) & :=\left\{\mathbf{X} \in \mathcal{D}(b)^{n} \mid D^{\prime}(\mathbf{X})=\left(s_{1}, \cdots, s_{k}\right)\right\}
\end{aligned}
$$

(2)' (tilde' order and tilde' descent)

We denote by $x=(j, r) \in \mathcal{D}(b)$ if

$$
x=j p+r \in \mathcal{D}(b), \quad j= \begin{cases}0,1, \cdots, c-1 & (r=0,1, \cdots, p-2) \\ 0,1, \cdots, c-2 & (r=p-1)\end{cases}
$$

and we set an order $\prec^{\prime}$ on $\mathcal{D}(b)$ as follows.

$$
\begin{aligned}
& (0,0) \prec^{\prime}(1,0) \prec^{\prime} \cdots \prec^{\prime}(c-1,0) \prec^{\prime} \\
& (0,1) \prec^{\prime}(1,1) \prec^{\prime} \cdots \prec^{\prime}(c-1,1) \prec \\
& (0,2) \prec^{\prime}(1,2) \prec^{\prime} \cdots \prec^{\prime}(c-1,2) \prec \\
& \prec^{\prime} \cdots \prec^{\prime} \\
& (0, p-1) \prec^{\prime}(1, p-1) \prec^{\prime} \cdots \prec^{\prime}(c-2, p-1)
\end{aligned}
$$

We say that $\mathbf{A}=\left(A_{1}, \cdots, A_{n}\right) \in \mathcal{D}(b)^{n}$ has tilde'-descent at $k \in[n]$ if and only if (i) $A_{k} \succ^{\prime} A_{k+1}(k=1,2, \cdots, n-1)$, and (ii) $A_{n} \equiv p-1(\bmod p)$.

The tilde'-descent set and the set of A's with given tilde'-descent set are defined by

$$
\begin{aligned}
\widetilde{D}^{\prime}(\mathbf{A}) & :=\{k \in[n] \mid \mathbf{A} \text { has a tilde'-descent at } k \in[n]\}, \quad \mathbf{A} \in \mathcal{D}(b)^{n} \\
\widetilde{\mathcal{D}}_{b}^{\prime}\left(s_{1}, \cdots, s_{k}\right) & :=\left\{\mathbf{A} \in \mathcal{D}(b)^{n} \mid \widetilde{D}^{\prime}(\mathbf{A})=\left(s_{1}, \cdots, s_{k}\right)\right\}
\end{aligned}
$$

Since $\sigma \in G_{p, n}$ has d'-descent at $n$ if and only if the corresponding $\mathbf{X} \in$ $\mathcal{D}_{b}\left(s_{1}, \cdots, s_{k}\right)$ satisfies $X_{n}>b-c$, the subgraph $\mathcal{L}$ of the square lattice is 
replaced by $\mathcal{L}^{\prime}$ given below.

$\mathcal{L}^{\prime}=(\{0,1, \cdots, n-1\} \times\{0,1, \cdots, b-1\}) \cup\{(n, j) \mid j=0,1, \cdots, b-c\}$

and thus, if $s_{k}=n$, the last column in the determinant is replaced by

$$
\left(\begin{array}{c}
n-s_{i}+b-1 \\
b-1
\end{array}\right)-\left(\begin{array}{c}
n-s_{i}+b-c \\
b-c
\end{array}\right), \quad 0 \leq i \leq k-1
$$

\section{Appendix}

\subsection{Generalized Carries Process}

In this subsection we consider a generalized carries process studied in [11, 12] called $(b, n, p)$-carries process, and present a simple way for diagonalizing the transition probability matrix. To discuss two cases $(p=1$ or $p \neq 1)$ simultaneously, we introduce the following parameter.

$$
\ell:=\ell(p)=\left\{\begin{array}{cc}
n & (p \neq 1) \\
n-1 & (p=1)
\end{array}\right.
$$

Let $p \in \mathbf{Q} \cap[1, \infty)$ and $\mathcal{C}(p):=\{0,1, \cdots, \ell(p)\} .( \pm b, n, p)$-carries process are the Markov chains on $\mathcal{C}(p)$ whose random mapping representations are given below respectively.

\section{Definition 3.1.}

(1) $(+b, n, p)$-carries process : let $b=p c+1 \in \mathbf{N}, c \in \mathbf{N}$. Given the carry $C_{k}^{+} \in \mathcal{C}(p)$ from the preceding digit, choose $X_{1}, \cdots, X_{n} \in \mathcal{D}(b)$ uniformly at ramdom, so that the carry $C_{k+1}^{+} \in \mathcal{C}(p)$ to the next digit is determined by the following equation.

$$
C_{k}^{+}+X_{1}+\cdots+X_{n}+\frac{b-1}{p^{*}}=C_{k+1}^{+} b+r, \quad r \in \mathcal{D}(b)
$$

where $\frac{1}{p}+\frac{1}{p^{*}}=1$.

(2) $(-b, n, p)$-carries process : let $b=p c-1 \in \mathbf{N}$ and $c \in \mathbf{N}$. Given the carry $C_{k}^{-} \in \mathcal{C}(p)$ from the preceding digit, choose $X_{1}, \cdots, X_{n} \in \mathcal{D}(b)$ uniformly at random, so that the carry $C_{k+1}^{-} \in \mathcal{C}(p)$ to the next digit is given by the following equation.

$$
C_{k}^{-}+X_{1}+\cdots+X_{n}+\frac{b+1}{p}-1=\left(n-C_{k+1}^{-}\right) b+r, \quad r \in \mathcal{D}(b) .
$$


The origin of those processes is as follows; if we consider the base $( \pm b)$-expansion of integers using $\mathcal{D}(b ; a):=\{a, a+1, \cdots, a+b-1\}(a \in$ Z) as the digit set, then after some change of variables the corresponding carries processes become $( \pm b, n, p)$-carries process [11, 12]. Let $P_{c}^{ \pm}:=$ $\left\{P_{c}^{ \pm}(i, j)\right\}_{i, j=0,1, \cdots, \ell}, P_{c}^{ \pm}(i, j):=P\left(C_{k+1}^{ \pm}=j \mid C_{k}^{ \pm}=i\right)$ be the transition probability matrix. Then

$$
\begin{aligned}
& P_{c}^{ \pm}(i, j)=b^{-n}\left[x^{A^{ \pm}(i, j)}\right]\left(\frac{1-x^{b}}{1-x}\right)^{n+1}, \\
& A^{+}(i, j):=j b-i+\frac{b-1}{p}, \quad A^{-}(i, j):=-i+(n-j+1) b-\frac{b+1}{p}
\end{aligned}
$$

where we denote by $\left[x^{k}\right] f$ the coefficient of $x^{k}$ in the power series $f$. Moreover we set $(\ell+1) \times(\ell+1)$-matrices $V=\left\{v_{i j}\right\}_{i, j=0, \cdots, \ell}, U=\left\{u_{i j}\right\}_{i, j=0, \cdots, \ell}$ whose elements are given respectively by

$$
\begin{aligned}
v_{i j} & :=\left[x^{j}\right]\left((1-x)^{n+1} A_{n-i}(x)\right), \text { where } A_{m}(x):=\sum_{k=0}^{\infty}(p k+1)^{m} x^{k} \\
u_{i j} & :=\left[x^{n-j}\right]\left(\begin{array}{c}
n+\frac{x-1}{p}-i \\
n
\end{array}\right) .
\end{aligned}
$$

Further, let $U=\left(\mathbf{u}_{0}, \cdots, \mathbf{u}_{\ell}\right), V=\left(\begin{array}{c}\mathbf{v}_{0} \\ \vdots \\ \mathbf{v}_{\ell}\end{array}\right)$ be the columm vector (resp. row vector) representation of $U$ (resp. $V$ ). We give a simple argument to diagonalize $P_{c}^{ \pm}$which uses the fact that the $N$-composition of $( \pm b, n, p)$ carries proceesses has the same distribution as that of $\left(( \pm b)^{N}, n, p\right)$-carries process [12].

\section{Theorem 3.2.}

$$
\left(P_{c}^{ \pm}\right)^{N}=\sum_{k=0}^{\ell}( \pm b)^{-k N} E_{k}, \quad N=0,1, \cdots
$$

where $E_{k}$ is a projection matrix given by

$$
E_{k}=\left|\mathbf{u}_{k}\right\rangle\left\langle\mathbf{v}_{k}\right|, \quad \text { that is } \quad\left(E_{k}\right)_{i j}:=u_{i k} \cdot v_{k j} .
$$

Therefore $P_{c}^{ \pm}$is diagonalizable and the eigenvalues are $\left\{( \pm b)^{-k}\right\}_{k=0}^{\ell}$ respectively with right and left eigenvectors being $\mathbf{u}_{k}, \mathbf{v}_{k}$ for both processes. 
Proof. By definition, $P_{c}^{ \pm}(i, j)$ is equal to

$$
\begin{aligned}
P_{c}^{ \pm}(i, j) & =b^{-n} \sum_{r}\left[x^{b r}\right]\left(1-x^{b}\right)^{n+1} \cdot\left[x^{A^{ \pm}(i, j)-b r}\right] \frac{1}{(1-x)^{n+1}} \\
& =b^{-n} \sum_{r}\left[x^{r}\right](1-x)^{n+1} \cdot\left(\begin{array}{c}
n+A^{ \pm}(i, j)-b r \\
n
\end{array}\right) .
\end{aligned}
$$

(1) $(+b)$-case : we have

$$
A^{+}(i, j)-b r=\frac{b\{p(j-r)+1\}-1}{p}-i .
$$

since $A^{+}(i, j)-b r \in \mathbf{Z}$, in order that the binomial coefficient in RHS in (3.2) is nonzero, it is necessary that $b\{p(j-r)+1\}-1 \geq 0$ which is equivalent to $j-r \geq-\frac{b-1}{p} \cdot \frac{1}{b}=-c \cdot \frac{1}{b}$. Since $c=\frac{b-1}{p} \in\{1,2, \cdots, b-1\}$ which implies $-1 \leq-c \cdot \frac{1}{b} \leq 0$, this condition is equivalent to $r \leq j$. By the definition of $u_{i j}$ and by (3.3),

$$
\left(\begin{array}{c}
n+A(i, j)-b r \\
n
\end{array}\right)=\sum_{k} u_{i k}(b\{p(j-r)+1\})^{n-k} \cdot 1(r \leq j) .
$$

Substituting this equation to (3.2) yields

$$
P_{c}^{+}(i, j)=\sum_{k} b^{-k} u_{i k} \sum_{r=0}^{j}\left[x^{r}\right](1-x)^{n+1} \cdot\left[x^{j-r}\right] A_{n-k}(x)=\sum_{k} b^{-k} u_{i k} \cdot v_{k j} .
$$

Since $N$-composition of $( \pm b, n, p)$-carries has the same distribution as that of $\left(( \pm b)^{N}, n, p\right)$-carries process [12, replacing $b$ by $b^{N}$ in (3.4) yields the expression for $\left(\left(P_{c}^{ \pm}\right)^{N}\right)_{i j}$ for $N \geq 1$. For $N=0$, we put $b=1$ in (3.4) and note that LHS is equal to $\delta_{i j}$.

(2) $(-b)$-case : we have

$$
A^{-}(i, j)-b r=\frac{\{p(n+1-j-r)-1\} b-1}{p}-i .
$$

In order that the binomial coefficient in (3.2) is nonzero, it is necessary that $\{p(n+1-j-r)-1\} b-1 \geq 0$ which is equivalent to

$$
n+1-j-r \geq \frac{b+1}{p} \cdot \frac{1}{b}=\frac{c}{b} .
$$


Noting that $c \in\{1,2, \cdots, b\}$ for $p>1$ and $c=b+1$ for $p=1,(3.6$ ) is equivalent to the condition $j+r \leq \ell$. By definition of $u_{i j}$, and by (3.5), we have

$$
\left(\begin{array}{c}
n+A^{-}(i, j)-b r \\
n
\end{array}\right)=\sum_{k} u_{i k}(\{p(n+1-j-r)-1\} b)^{n-k} \cdot 1(j+r \leq \ell)
$$

and substituting this equation into (3.2) and change of variables $s=n+1-r$ yields

$$
\begin{aligned}
& P_{c}^{-}(i, j) \\
= & b^{-n} \sum_{s}\left[x^{n+1-s}\right](1-x)^{n+1} \sum_{k} u_{i k}[\{p(s-j)-1\} b]^{n-k} \cdot 1(j+n+1-s \leq \ell) \\
= & b^{-n} \sum_{s}(-1)^{n+1}\left[x^{s}\right](1-x)^{n+1} \sum_{k} u_{i k}\{p(j-s)+1\}^{n-k}(-1)^{n-k} b^{n-k} \cdot 1(j+1 \leq \ell-n+s) \\
= & -\sum_{k}(-b)^{-k} u_{i k} \sum_{s}\left[x^{s}\right](1-x)^{n+1} \cdot\{p(j-s)+1\}^{n-k} 1\left(\begin{array}{ll}
j+1 \leq s & (p \neq 1) \\
j+2 \leq s & (p=1)
\end{array}\right) .
\end{aligned}
$$

When $p=1$ and $s=j+1$, we have $\{p(j-s)+1\}^{n-k}(-1)^{n-k}=0$ so that the indicator function in RHS may be simplified to $1(j+1 \leq s)$ for both cases. Therefore for any $p$,

$P_{c}^{-}(i, j)=-\sum_{k}(-b)^{-k} u_{i k} \sum_{s}\left[x^{s}\right](1-x)^{n+1}\{p(j-s)+1\}^{n-k} 1(j+1 \leq s)$.

Since we have

$$
\sum_{s=0}^{n+1}\left[x^{s}\right](1-x)^{n+1}\{p(j-s)+1\}^{n-k}=0, \quad k=0,1, \cdots, n,
$$

the indicator function $1(j+1 \leq s)$ in RHS in (3.7) may be replaced by $-1(s \leq j)$. Hence we have

$P_{c}^{-}(i, j)=\sum_{k}(-b)^{-k} u_{i k} \sum_{s}\left[x^{s}\right](1-x)^{n+1}\{p(j-s)+1\}^{n-k} 1(s \leq j)=\sum_{k}(-b)^{-k} u_{i k} v_{k j}$.

The rest of the proof is the same as that of $(+b)$-case. $\square$ 
Next, we present a result on the relation between the $( \pm b, n, p)$-carries process and $(b, n, p)$-shuffle [12], which is a generalization of those in [3, 4] 2. Let $S$ be the $(b, n, p)$-shuffle and let $R: \sigma=\left\{\left(\sigma(i), \sigma_{c}(i)\right)\right\}_{i=1}^{n} \mapsto \sigma^{\prime}:=$ $\left\{\left(\sigma(i), p-\sigma_{c}(i)\right)\right\}$ be the color-reversing map. Let $\{\sigma(k)\}_{k=0}^{\infty}$ be the random walk on $G_{p, n}$ by applying the $(b, n, p)$-shuffle repeatedly to the identity :

$$
\sigma(k):=S^{k} \circ \sigma(0), \quad \sigma(0):=i d \in G_{p, n} .
$$

Moreover, as a variant of that, let $\left\{\sigma^{\prime}(k)\right\}_{k=0}^{\infty}$ be another random walk on $G_{p, n}$ applying $R$ in even steps :

$$
\sigma^{\prime}(k):= \begin{cases}S \circ \sigma^{\prime}(k-1) & (k: \text { odd }) \\ R \circ S \circ \sigma^{\prime}(k-1) & (k: \text { even })\end{cases}
$$

Theorem 3.3. [12] Let $p \in \mathbf{N}$.

(1) $b \equiv 1(\bmod p)$ :

$$
\{d(\sigma(k))\}_{k=0}^{\infty} \stackrel{d}{=}\left\{C_{k}^{+}\right\}_{k=0}^{\infty} .
$$

(2) $b \equiv-1(\bmod p):$ let $d(k):=\left\{\begin{array}{ll}n-d^{\prime}\left(\sigma^{\prime}(k)\right) & (k: \text { odd }) \\ d\left(\sigma^{\prime}(k)\right) & (k: \text { even })\end{array}\right.$. Then $\{d(k)\}$ is a Markov chain with

$$
\{d(k)\}_{k=0}^{\infty} \stackrel{d}{=}\left\{C_{k}^{-}\right\}_{k=0}^{\infty} .
$$

\subsection{Some Relation between $(b, n, p)$-carries process and $(b, n, p)$-shuffle}

In this subsection we assume $b \equiv 1(\bmod p)$ and we present a result that the eigenspace and its multiplicity of the transition probability matrix $P_{s}$ of $\{\sigma(k)\}$ can be represented by that of the right eigenvectors of $(b, n, p)$-carries process. This fact has been discussed in several papers (e.g., [5]) for $p=1$. In $§ 3.2 .3$, we briefly state corresponding results for $b \equiv-1(\bmod p)$.

\footnotetext{
${ }^{2}$ we would like to take this opportunity to correct the statement in [12] for $(-b)$-case.
} 


\subsubsection{Decent Algebra}

We collect some preparatory facts on the descent algebra. The following fact is the $G_{p, n}$-version of Gessel's lemma, proved in [12].

Lemma 3.4. For each $\sigma \in G_{p, n}$, let

$$
c_{i j}^{\sigma}:=\sharp\{(\mu, \tau) \mid d(\mu)=i, d(\tau)=j, \mu \tau=\sigma\}, \quad i, j=0,1, \cdots, \ell
$$

(1) The generating function of $\left\{c_{i j}^{\sigma}\right\}_{i, j}$ is given by

$$
\sum_{i, j \geq 0} c_{i j}^{\sigma} \cdot \frac{s^{i}}{(1-s)^{n+1}} \frac{t^{j}}{(1-t)^{n+1}}=\sum_{a, b \geq 0}\left(\begin{array}{c}
n+p a b+a+b-d(\sigma) \\
n
\end{array}\right) s^{a} t^{b} .
$$

(2) $d\left(\sigma_{1}\right)=d\left(\sigma_{2}\right)$ implies $c_{i j}^{\sigma_{1}}=c_{i j}^{\sigma_{2}}$ so that for each $k \in\{0,1, \cdots, \ell\}$ we take $\sigma \in G_{p, n}$ with $d(\sigma)=k$ and set

$$
c_{i j}^{k}:=c_{i j}^{\sigma}
$$

(3) $c_{i j}^{k}=c_{j i}^{k}$.

We consider the descent algebra and its inverse involution :

$$
D_{i}:=\sum_{d(\sigma)=i} \sigma \in \mathbf{C}\left[G_{p, n}\right], \quad \Theta_{i}:=\sum_{d\left(\sigma^{-1}\right)=i} \sigma \in \mathbf{C}\left[G_{p, n}\right], \quad i=0,1, \cdots, \ell .
$$

Then by Lemma 3.4

Lemma 3.5. (1) $D_{i} D_{j}=\sum_{k} c_{i j}^{k} D_{k}$ (2) $D_{i} D_{j}=D_{j} D_{i}$ (3) $\left\{\Theta_{i}\right\}$ satisfy the same properties.

\subsubsection{Eigenvalues and eigenspaces of the $(b, n, p)$-shuffle}

We define $e_{0}, e_{1}, \cdots, e_{\ell} \in \mathbf{C}\left[G_{p, n}\right]$ by the following equation.

$$
S:=\sum_{\sigma \in G_{p, n}} P((b, n, p) \text {-shuffle }=\sigma) \sigma=\frac{1}{b^{n}} \sum_{i=0}^{\ell}\left(\begin{array}{c}
n+\frac{b-1}{p}-i \\
n
\end{array}\right) \Theta_{i}=\sum_{k=0}^{\ell} b^{-k} e_{\ell-k} .
$$


In other words, $e_{\ell-k}:=\left[b^{n-k}\right]\left(b^{n} S\right)$. And we also define $f_{0}, f_{1}, \cdots, f_{\ell} \in$ $\mathbf{C}\left[G_{p, n}\right]$ as the inverse counterparts :

$T:=\sum_{\sigma \in G_{p, n}} P\left(((b, n, p) \text {-shuffle })^{-1}=\sigma\right) \sigma=\frac{1}{b^{n}} \sum_{i=0}^{\ell}\left(\begin{array}{c}n+\frac{b-1}{p}-i \\ n\end{array}\right) D_{i}=\sum_{k=0}^{\ell} b^{-k} f_{\ell-k}$.

The folowing proposition is essentially due to [10], which implies that $\left\{\Theta_{i}\right\}$ and $\left\{e_{\ell-k}\right\}$ are related via the right eigenvectors of $P_{c}^{ \pm}$.

\section{Proposition 3.6.}

$$
\Theta_{i}=\sum_{k=0}^{\ell} v_{k i} e_{\ell-k}, \quad(2) \quad e_{\ell-k}=\sum_{i=0}^{\ell} u_{i k} \Theta_{i}
$$

Similarly,
(3)

$$
D_{i}=\sum_{k=0}^{\ell} v_{k i} f_{\ell-k}
$$
(4) $f_{\ell-k}=\sum_{i=0}^{\ell} u_{i k} D_{i}$

Proof. By eq. (3.8) we have

$$
\sum_{i=0}^{\ell}\left(\begin{array}{c}
n+\frac{b-1}{p}-i \\
n
\end{array}\right) \Theta_{i}=\sum_{k=0}^{\ell} b^{n-k} e_{\ell-k}
$$

which is a polynomial in $b$. Taking the coefficient of $b^{n-k}$ on both sides yields (2). (1) follows from $V=U^{-1}$. (3) and (4) follow similarly.

Now we have a representation of the eigenspace of the transition probability matrix of $\{\sigma(k)\}$.

\section{Theorem 3.7.}

(1) Let $P_{s}=\left\{P_{s}(\mu, \tau)\right\}_{\mu, \tau}, P_{s}(\mu, \tau):=\mathbf{P}(\sigma(k+1)=\tau \mid \sigma(k)=\mu), \mu, \tau \in$ $G_{p, n}$ be the transition probability matrix of $\{\sigma(k)\}$. Then the set of eigenvalues of $P_{s}$ is $\left\{b^{-j}\right\}_{j=0}^{\ell}$ and with left (resp. right) eigenspace being Range $L\left(e_{\ell-j}\right)$ (resp. Range $L\left(f_{\ell-j}\right)$ ), where $L(A)$ is the left regular representation of $A \in \mathbf{C}\left(G_{p, n}\right)$.

(2) The multiplicity of the eigenvalue $b^{-j}$ is equal to $u_{0 j} p^{n} n$ !, which is equal to the Stirling - Frobenius cycle number. 
Proof. (1) Since the $N$-composition of $(b, n, p)$-shuffles has the same distribution as that of $\left(b^{N}, n, p\right)$-shuffle (Lemma 3.9), we have

$$
S^{N}=\frac{1}{b^{N n}} \sum_{i=0}^{l}\left(\begin{array}{c}
n+\frac{b^{N}-1}{p}-i \\
n
\end{array}\right) \Theta_{i}=\sum_{k=0}^{l}\left(\frac{1}{b^{k}}\right)^{N} e_{\ell-k}, \quad N=0,1, \cdots,
$$

The statement for the eigenvalues and left eigenspaces follows by taking the left regular representation of both sides of (3.9). For the right eigenspace, we consider the inverse shuffle $T$.

(2) By (3.9),$L\left(e_{\ell-j}\right)$ is a projection so that dim Range $L\left(e_{\ell-j}\right)=$ trace $L\left(e_{\ell-j}\right)$. Since

$$
\operatorname{trace} L\left(e_{\ell-j}\right)=\operatorname{trace}\left(\sum_{i=0}^{l} u_{i j} L\left(\Theta_{i}\right)\right)=\sum_{i=0}^{l} u_{i j} \operatorname{trace}\left[L\left(\Theta_{i}\right)\right] \text {, }
$$

and since trace $\left[L\left(\Theta_{i}\right)\right]=\left|G_{p, n}\right| 1(i=0)$, we have

$$
\sum_{i=0}^{l} u_{i j} \operatorname{trace}\left[L\left(\Theta_{i}\right)\right]=u_{0 j} \operatorname{trace}\left(L\left(\Theta_{0}\right)\right)=u_{0 j} p^{n} n !
$$

\subsubsection{In the case of $b \equiv-1(\bmod p)$}

In this subsection we set $b=p c-1 \equiv-1(\bmod p), c \in \mathbf{N}$. Then some of the previous discussion still work from which we conclude that the transition probability matrix $P_{s}$ can not be diagonalized. Since the argument is parallel as that of previous ones, we omit the detail and state the results only. We first give a formula for the probability to obtain a given $\sigma$ as $(b, n, p)$-shuffle. As before $R \circ \sigma$ is the reverse of colors of $\sigma \in G_{p, n}$. For simplicity, we write "( $(b, n, p)$ " instead of " $(b, n, p)$-shuffle".

Lemma 3.8. Let $\sigma \in G_{p, n}$.

(1) $b=p c+1$

$$
P((b, n, p)=\sigma)=b^{-n}\left(\begin{array}{c}
n+c-d\left(\sigma^{-1}\right) \\
n
\end{array}\right) .
$$


(2) $b=p c-1$

$$
P(R \circ(b, n, p)=\sigma)=b^{-n}\left(\begin{array}{c}
n+c-1-d^{\prime}\left(\sigma^{-1}\right) \\
n
\end{array}\right) .
$$

We next study the composition of $(b, n, p)$-shuffles.

Lemma 3.9. [12] Let $b_{1}, b_{2} \equiv \pm 1(\bmod p)$.

$$
\left(b_{1}, n, p\right) \circ\left(b_{2}, n, p\right) \stackrel{d}{=}\left\{\begin{array}{lll}
\left(\left(b_{1} \cdot b_{2}\right), n, p\right) & \left(b_{2} \equiv 1 \quad(\bmod p)\right) \\
R \circ\left(\left(b_{1} \cdot b_{2}\right), n, p\right) & \left(b_{2} \equiv-1\right. & (\bmod p))
\end{array}\right.
$$

Lemma 3.10. $N$-composition of $(b, n, p)$ - shuffle has the same distribution to

$$
\overbrace{(b, n, p) \circ \cdots \circ(b, n, p)}^{N} \stackrel{d}{=} \begin{cases}\left(b^{N}, n, p\right) & (N: \text { odd }) \\ R \circ\left(b^{N}, n, p\right) & (N: \text { even })\end{cases}
$$

By using above lemmas, we have a formula for the $N$-composition of the element in $\mathbf{C}\left[G_{p, n}\right]$ corresponding to $(b, n, p)$-shuffle.

Proposition 3.11. Let

$$
S:=\sum_{\sigma} P((b, n, p)=\sigma) \sigma \in \mathbf{C}\left(G_{p, n}\right) .
$$

Then the $N$-composition of $S$ is given by

$$
S^{N}= \begin{cases}\sum_{k}\left(b^{-k}\right)^{N} \sum_{i} w_{i k} R \circ \Theta_{i}^{\prime} & (N: \text { odd }) \\ \sum_{k}\left(b^{-k}\right)^{N} \sum_{i} u_{i k} R \circ \Theta_{i} & (N: \text { even })\end{cases}
$$

where we set

$$
\Theta_{i}:=\sum_{\sigma: d\left(\sigma^{-1}\right)=i} \sigma, \quad \Theta_{i}^{\prime}:=\sum_{\mu: d^{\prime}\left(\mu^{-1}\right)=i} \mu, \quad w_{i k}:=\left[x^{n-k}\right]\left(\begin{array}{c}
n+\frac{x+1}{p}-1-i \\
n
\end{array}\right) .
$$

If $P$ were diagonalizable, we would have

$$
\sum_{i} w_{i k} R \circ \Theta_{i}^{\prime}=\sum_{i} u_{i k} R \circ \Theta_{i}
$$

for all $k$ which is not true except $k=0$. 


\subsection{Cut off for $(b, n, p)$-shuffle}

In this subsection we discuss the convergence speed of $\{\sigma(k)\}$ to the stationary distribution. Let $Q^{k}(\mu):=P(\sigma(k)=\mu)$ be the distribution of $\{\sigma(k)\}$, let $\pi$ be the uniform distribution on $G_{p, n}$, and let $\|\cdot\|_{T V}$ be the total variation distance. Since the mixing time of $\{\sigma(k)\}$ is in the order of $\frac{3}{2} \log _{b} n$, we set $k=\frac{3}{2} \log _{b} n+j$ to observe the asymptotic behavior of $\left\|Q^{k}-\pi\right\|_{T V}$ near the mixing time for large $n$. By a similar argument as that in [1], we have the following asymptotics, implying that the distance of $Q^{k}$ from the stationary distribution changes quickly near the mixing time.

Theorem 3.12. Setting $k=\frac{3}{2} \log _{b} n+j$ we have

$$
\begin{aligned}
\left\|Q^{k}-\pi\right\|_{T V} & =1-2 \Phi\left(-\frac{p}{4 \sqrt{3}} \cdot b^{-j}\right)+O\left(n^{-\frac{1}{4}}\right) \\
& \sim\left\{\begin{array}{cc}
1-\exp \left(-\frac{p^{2}}{2 \cdot 4^{2} \cdot 3} \cdot b^{-2 j}\right) & (j \rightarrow-\infty) \\
\frac{p}{2 \sqrt{6 \pi}} \cdot b^{-j} & (j \rightarrow \infty)
\end{array}\right. \\
\text { where } & \Phi(x):=\frac{1}{\sqrt{2 \pi}} \int_{-\infty}^{x} e^{-\frac{t^{2}}{2}} d t .
\end{aligned}
$$

This work is partially supported by JSPS KAKENHI Grant Number 20K03659(F.N.)

\section{References}

[1] Bayer, D., and Diaconis, P., : Trailing the dovetail shuffle to its lair, Ann. Appl. Prob. (1992)Vol. 2, No.2, 294-313.

[2] Borodin, A., Diaconis, P., and Fulman, J., On adding a list of numbers (and other one-dependent determinantal processes), Bull. Amer. Math. Soc. 47(4)(2010), 639-670.

[3] Diaconis, P., Fulman, J., Carries, shuffling, and an amazing matrix, The American Mathematical Monthly, 116(9)(2009), 780-803.

[4] Diaconis, P., Fulman, J., Carries, shuffling, and symmetric functions, Advances in Applied Mathematics, 43(2)(2009), 176-196. 
[5] Diaconis, P., Fulman, J., Foulkes characters, Eulerian idempotents, and an amazing matrix, J. Alg. Comb. 36(3)(2012), 425-440.

[6] Gessel, I., and Viennot, G., Binomial determinants, paths, and hook length formulae, Adv. Math. 58(3)(1985), 300-321.

[7] Holte, J., Carries, combinatorics, and an amazing matrix, The American Mathematical Monthly, 104(2)(1997), 138-149.

[8] Lindeström, B., on the vector representation of induced matroids. Bull. London Math. Soc. 5(1973), 85-90.

[9] MacMahon, P. A., Combinatory analysis. Vols. I, II (1915, 1916), Dover Publ.

[10] Miller, A., Foulkes characters for complex reflection groups, Proc. Amer. Math. Soc. 143(2015), no.8, 3281-3293.

[11] Nakano, F., and Sadahiro, T., A generalization of carries process and Eulerian numbers, Adv. Appl. Math. 53 (2014), 28 - 43.

[12] Nakano, F., and Sadahiro, T., A generalization of carries process and riffle shuffles, Disc. Math., 339 (2016), 974-991.

[13] Reiner, V., Signed permutation statistics, European J. Combin. 14(1993), $553-567$. 